\title{
ASTROMETRIC INTERFEROMETRY - CAN IT ESTABLISH A FUNDAMENTAL SYSTEM?
}

\author{
G.H. KAPLAN \\ U. S. Naval Observatory \\ Washington, D.C. 20392 \\ USA
}

\begin{abstract}
The astrometric optical interferometer on Mt. Wilson is providing a new source of astrophysical and astrometric data on bright stars. The instrument, with 12-meter baselines, has been in operation since late 1986. The interferometer is capable of wide-angle astrometry, that is, the determination of very precise stellar positions within a reference frame defined by bright stars spread across a large area (of order one steradian) of the sky. This paper addresses the question of whether such an instrument can be used to establish a fundamental system - that is, one tied to the Earth in some well-defined way. Some astrometric data from this instrument are presented to illustrate the difficulties involved. Proposed means of addressing these problems in future instruments are discussed.
\end{abstract}

\section{Introduction}

This paper expands upon some ideas which followed from a discussion in August 1988, among those of us involved with the Mt. Wilson astrometric optical interferometer project, concerning the "precision" and "accuracy" of the astrometric results from the interferometer. It explores in some detail a concern expressed by Michael Shao about the validity of the astrometric results since the interferometer's baselines did not appear to co-rotate with the Earth. I am considering here only the overall geometry of the interferometer observations and what its implications are for that type of instrument. The results may apply to other types of Earth-based, "fundamental" instruments transit circles, astrolabes, zenith tubes - but a more general discussion is beyond the current scope and my own expertise.

Here, I use the terms "absolute" or "fundamental" to describe star coordinates measured with respect to the Earth's instantaneous rotation axis: declinations and relative right ascensions. I will not deal with the origin of right ascension, i.e., the location of the equinox. I use the word "axis" frequently, and it should be considered synonymous with "angular velocity vector", that is, both an orientation and a magnitude (spin rate) are involved. Two axes are coincident only if both their orientation and magnitude are identical.

I wish to say at the outset that I do not mean to imply that observations which are not fundamental are not useful. In fact, astrometric observations from space (e.g., HIPPARCOS) will not be fundamental in the usual sense. However, if we are planning to eventually replace transit circles with interferometers we need to consider all the ramifications. As we enter an era where the most accurate wide-angle astrometry may be non-fundamental, we will need to pay much more attention to exactly 
how our coordinate systems are both defined and realized.

\section{The Mt. Wilson Optical Interferometer}

The optical interferometer on Mt. Wilson, near Los Angeles, is unfamiliar to many in the astrometric community. A significant amount of observational data has already been obtained from this unique instrument, which is essentially a long-baseline Michelson stellar interferometer. Stellar fringes were first detected in September 1986, and routine observational programs in stellar diameters and astrometry were begun in July 1987. The interferometer has been a joint project of the Smithsonian Astrophysical Observatory, the Naval Research Laboratory, the Massachusetts Institute of Technology, and the U. S. Naval Observatory. Key people involved in the development of this system include Michael Shao, Mark Colavita, Kenneth Johnston, Richard Simon, David Mozurkewich, Donald Hutter, James Hughes, John Hershey, John Pohlman, Braden Hines, and David Staelin.

The Mt. Wilson interferometer is a highly automated, rapid-response, wideband system which combines and interferes starlight from a pair of independently-moving $25-\mathrm{cm}$ siderostats. Currently, the instrument can alternate between pairs of siderostats forming two baselines, each 12 meters long. The instrument actively tracks a star's "white light fringe" in real time and has been designed specifically for absolute astrometry. The astrometric capabilities of this instrument were demonstrated on a limited data set from observations taken in the autumn of 1986 when only one baseline was available (Mozurkewich et al. 1988). Since that time, the second baseline has become operational, a number of instrumental systems have been improved, and experiments have begun with two-color observations. The latter is a means for greatly reducing the effects of the Earth's atmosphere on the observations. More technical detail on this instrument may be found in Shao et al. (1988), Colavita et al. (1987), Shao (1988), and Kaplan et al. (1988).

When one-color data from a half dozen nights of observations are combined, the formal errors in position for each star have been generally in the range 15-25 milliarcseconds in declination and somewhat worse in right ascension. The two-color technique promises to reduce these formal uncertainties to perhaps a few milliarcseconds. The current instrument is limited to stars brighter than about visual magnitude 5 and sky coverage is restricted to declinations between +15 and +55 degrees. However, this instrument has been sufficiently successful that the U. S. Naval Observatory is currently involved in the design and construction of a much larger astrometric optical interferometer to be completed by 1994 and located in the coastal mountains of central California.

Clearly we must understand exactly what our star positions mean; specifically, in what reference frame are they expressed and how can they be related to more conventional systems, for example, the FK5? Up to this point we have essentially been treating the Mt. Wilson optical data like VLBI data as far as our data reduction procedures are concerned. However, our data are unlike VLBI data in two ways: (1) we can observe only over part of a day (less than half of one rotation of the Earth), and (2) our baseline components vary significantly over the course of the observations. We have been aware of these distinctions for some time, and each of us involved in the project has expressed the situation in slightly different ways. We have made statements to the effect that "we have different baselines for different stars" or "for any given star, the baseline is a function of hour angle", or something similar. Given these realities, I reconsider here the statement frequently made by interferometry people (radio or optical) that "our declinations and relative right ascensions are 
absolute". Under what conditions is that true?

\section{Interferometers and the Earth's Rotation Axis}

Unlike transit circles, PZTs, and astrolabes, interferometers have no tie to the local gravity field and therefore have no well-defined latitude. Viewed from inertial space, an interferometer baseline is simply a vector which rotates around some axis once per sidereal day (see Figure 1). The only actual tie to the Earth is the assumption that the axis about which the baseline rotates is coincident with that of the Earth. A lot rests on that assumption, both for the VLBI/ Earth rotation community and for those of us interested in interferometric astrometry.

Regardless of whether the interferometer's axis does coincide with that of the Earth, one can always determine, from an appropriate set of observations, a number of parameters describing the baseline, b, and its apparent axis of rotation, $\mathbf{w}$. The $a$ priori star coordinates at the observing epoch are assumed to define an inertial coordinate system (at some level of accuracy) for these measurements. There are a number of ways of parameterizing this geometric information, some more useful than others.

The Earth rotation community, in giving very high weight to VLBI data, takes as axiomatic the coincidence of any

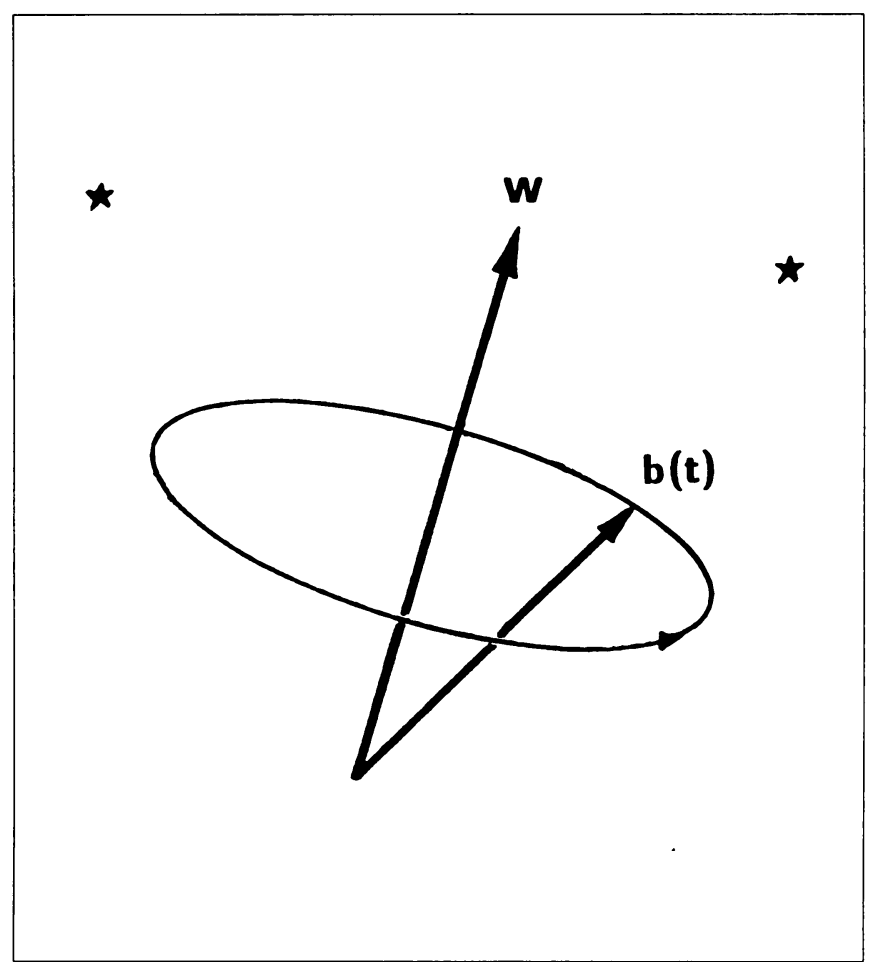

Figure 1. Diurnal motion of an interferometer baseline, $\mathbf{b}$, about its apparent axis $\mathbf{w}$.

VLBI axis w with that of the Earth. In fact, a stronger assumption is made: VLBI baselines are tied rigidly to the crust of the Earth. The latter assumption is a sufficient but not a necessary condition for the coincidence of the axes.

But Earth rotation studies are a special case of interferometric astrometry, and for those of us interested primarily in star or radio source positions, there is no requirement that the baselines be tied to the Earth's crust. Indeed, there is no need that the baselines in any way remain constant from one night to the next or even that the same baseline be used. However, it is necessary to assume that during the course of observations, the baseline's axis of rotation is the same as that of the Earth. The extent to which interferometrically determined star coordinates are fundamental rests entirely on the 
validity of the assumption that the baseline's apparent axis of rotation is coincident, in both orientation and magnitude, with that of the Earth.

The data indicate that the VLBI community is on firm ground in stating that their source positions are fundamental in the sense I am using here. The optical interferometer data are quite different. We know that the Mt. Wilson baselines do not remain constant during our observations. In our current data reduction approach, we solve for rates of change of the rectangular baseline components during the observations. Occasionally we also solve for the corresponding accelerations. We cannot avoid some kind of procedure like this; the data demand it. The tacit assumption is that solving for the baseline motion effectively reduces the baseline to a constant vector within our local Earth-fixed coordinate system, thus maintaining the fundamental nature of our instrument. By the time we published our original astrometry paper (Mozurkewich et. al. 1988), we had begun to recognize the subtle complications of what we were doing, and we couched our results in appropriately conservative terms. In the following, I explore these subtleties more fully. My thesis here is that, as long as we have to solve for baseline motions from the observations themselves, the observations are effectively related to an arbitrary axis of rotation. Although we can, in fact, locate that axis of rotation within the reference frame defined by the catalog of a priori star positions, by this point we have abandoned any frame of reference with any physically well-defined anchor. Any star positions obtained from such observations are not, therefore, fundamental.

\section{The Geometry of Offset Axes}

This section presents some geometrical results which are useful background information for this discussion.

Suppose a baseline's axis of rotation were not coincident with that of the Earth. Viewed from a truly Earth-fixed (topocentric) system, how would the baseline appear to move?

Consider a baseline $\mathbf{b}$, a constant vector in our usual Earth-fixed system: the $z$ axis points toward the north celestial pole, the $x y$ plane is the equator, and the $x z$ plane is the local meridian. The system, hence $\mathbf{b}$, rotates about the $z$ (polar) axis ai the sidereal rate. Viewed from an inertial frame, $b$ is the rotating vector $\mathbf{b}(t)$. Its axis of rotation, $\mathbf{w}$, coincides with that of the Earth. Now suppose we slightly tilt the Earth and its axis. The vector $b$ now rotates about a slightly offset axis, $\mathbf{w}^{\prime}$, again at the sidereal rate. Viewed from the inertial frame, $\mathbf{b}$ is now the rotating vector $\mathbf{b}^{\prime}(t)$. Form the difference vector $\mathbf{d}(t)=\mathbf{b}^{\prime}(t)-\mathbf{b}(t)$ (see Figure 2). What does the locus of $\mathbf{d}(t)$ look like, back in the Earth-fixed system where $b$ is a constant?

A little bit of algebra shows the following. In the Earth-fixed (topocentric) system the difference vector $\mathbf{d}(t)$ executes a small ellipse over the course of a day. The plane of the ellipse is orthogonal to $b$ (as we would expect, since we are only dealing with rotations here and no change of length should result). The major axis of the ellipse is in the plane formed by $b$ and the $z$ (polar) axis. The length of the semimajor axis is simply the angular offset of $\mathbf{w}^{\prime}$ from $\mathbf{w}$ (in radians) times the length of $b$. The ratio of minor to major axis is identical to the ratio of the $z$ (polar) component of the baseline to the total baseline length (that is, $\mathbf{b} \cdot \mathbf{w} / \mathbf{l b} \| \mathbf{w} \mid$ ). The instantaneous phase angle of $\mathbf{d}$ as it traces out this small ellipse depends on both the local sidereal time and the relative orientation of the two axes. The vector d, does, however, trace out the ellipse in a counterclockwise manner as viewed from the origin, 
and completes the circuit in one sidereal day.

If, in addition, the rotation rates are slightly different for the two axes, the ellipse becomes distorted in the direction of its minor axis (that is, orthogonal to the rotation axis). In this case the ellipse will not close on itself after one day.

How would an offset rotation of one of the optical interferometer's baselines appear in the topocentric Mt. Wilson reference frame? Assume a quarter-arcsecond angular separation of the direction of rotation axis of the baseline from that of the Earth (we shall see in section 5 that this is not too unreasonable a number). As seen from Mt. Wilson, the baseline would appear to undergo an elliptical oscillation over the course of a sidereal day. As stated above, the plane of the ellipse would be orthogonal to the baseline.

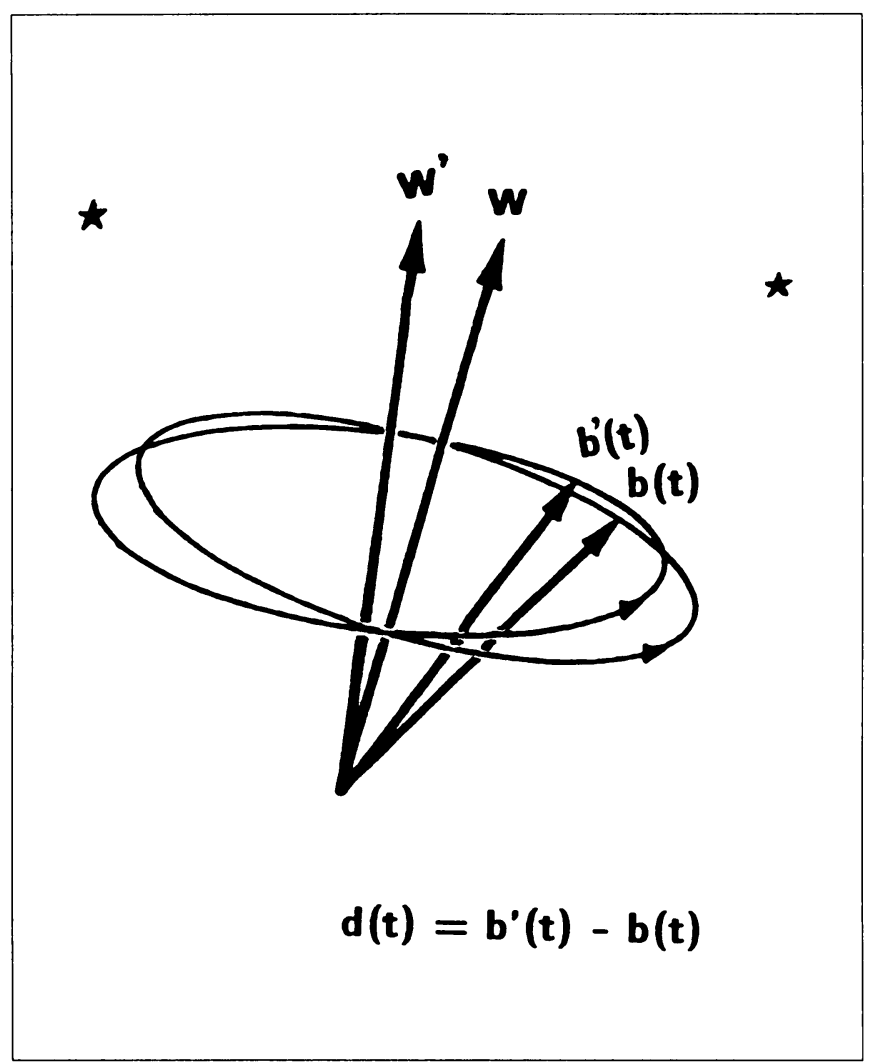

Figure 2. Comparison of diurnal motion of an interferometer baseline about two axes, w and Figure 1. Diurnal

For either of our 12-meter as-

trometric baselines the plane of the ellipse would obviously be vertical and, for the hypothesized quarter-arcsecond axis offset, the major axis would be 29 microns long. The oscillation would progress in a counterclockwise manner viewed from the south. For the south-north baseline, the plane of the ellipse would lie in a vertical east-west plane, with the major axis oriented vertically, and a minor-to-major axis ratio of 0.83 (eccentricity 0.56 ). For the south-east baseline, the ellipse would lie in a vertical plane oriented at an azimuth of 147 degrees, with the major axis tilted 51 degrees from vertical on a great circle passing through the celestial pole. The minor-to-major axis ratio would be 0.45 (eccentricity 0.89 ).

Suppose one of the baselines happened, by chance, to undergo the diumal counterclockwise elliptical oscillation described above. That motion, viewed from an inertial frame, would be equivalent to rotation about an axis offset from that of the Earth by a quarter arcsecond. The exact orientation of the offset axis - the position of the apparent celestial pole for that baseline - would depend on the phase angle of the baseline as it traces out its ellipse as a function of local sidereal time.

Now the geometry of this elliptical motion is specified fairly tightly. The probability of any 
baseline undergoing this kind of motion by chance over the course of an entire day is minuscule. However, we have no way of knowing what our baselines do over the course of an entire day, and we do not need to know. The only information we have on baseline motion, and the only information that is important, is derived from our observations, which rarely take up more than 0.4 day. Furthermore, the size of the ellipse and the phase angle of the oscillation are arbitrary. Therefore, any arbitrary baseline motion might simulate an arc of the ellipse over the span of observations. The condition for this to occur is that the motion be dominated by periodic components with periods of about a day or longer. Another condition, that the motion preserve baseline length, is actually not required since a "stretch" or "shrinkage" of the baseline can be considered to be an independent (orthogonal) effect.

The baseline motions that we have experienced with the Mt. Wilson instrument are definitely dominated by long-period components; most often we assume them to be linear over the course of a night, although on many nights that is clearly an oversimplification. We actually expect that thermal effects would generally be dominated by periodicities of one solar day.

It seems possible, then, that our nightly baseline motion might mimic the motion that would be equivalent to that from an axis of rotation offset from that of the Earth. If that is indeed so, then our instrumental system is not fundamental.

\section{An Experiment With a Real Night's Data}

I have performed some numerical experiments with a particularly good set of one-color observations taken on the night of 21 August 1987. This data set was chosen because it spans the entire night, the residuals are low (4 microns RMS, equivalent to about 70 milliarcseconds on the sky), and because the baseline drift on that night was obvious and fairly linear-looking on both baselines. That night we observed 32 FK5 stars, each several times at various hour angles on both baselines. Each observation consists of a recording of time, star and baseline identifier, and the measured optical path length difference between the two active siderostats (in VLBI terminology, the "delay", expressed in units of length). We pass the observations through two programs, CALC and SOLVE. CALC computes the a priori geometry of the observation from conventional models of precession, nutation, aberration, Earth rotation, atmosphere, etc., an assumed constant baseline vector, and FK5 star positions (the models are documented in Kaplan et al. 1989). CALC compares its computed path length difference with that actually measured, producing path length (O-C)s. SOLVE is a standard least-squares program for extracting various kinds of geometric information, including star positions, from CALC's (O-C)s.

Figure 3(a) shows the 21 August data for the SN (south $->$ north) baseline, where the baseline is treated as a constant over the course of the night. Time increases downward, and the horizontal axis represents post-solution residuals in optical path length, expressed in microns. The gaps in the data represent periods of time when the SE (south $\rightarrow$ east) baseline was in use. This is the simplest possible treatment of the data; star positions were not solved for (some of the scatter in the residuals is due to slightly incorrect star positions). Obviously treating the baseline as a constant is inadequate; the slope in the residuals amounts to about 40 microns over the course of the night for the SN baseline. The data for the SE baseline, which is not shown, show a slope of almost 20 microns (this was the "old" SE baseline, which was only 8 meters long).

Figure 3(b) shows the same data treated in the conventional way, that is, the three rectangular 
components of the baseline are assumed to vary linearly with time over the course of the night and the three rates of change for each baseline are solved for (the "linear-drift" model). The RMS of the residuals is 3.72 microns for the SN baseline data shown (4.22 for the SE baseline data).

Next, I added two parameters and their partials to the program SOLVE (all SOLVE parameters can be turned on or off at will). The parameters DELPSI and DELEPS are two small angles in the ecliptic system. (The fact that we have no sensitivity to the ecliptic is irrelevant for this purpose; we know well enough where it is on the sky and these are differential angles which we need to only about three significant digits.) These parameters provide the location of the baseline's apparent axis of rotation within the reference frame of the a priori star coordinates. The same parameters could yield information on precession/nutation modelling errors if the baselines were well-enough anchored in the Earth's crust so that we could assume that the baseline's apparent axis of rotation was coincident with that of the Earth. For the optical interferometer data, I cannot make that assumption; I simply want to know whether the baseline's motion - from whatever causes - can be modelled as equivalent to that from a slightly offset axis.

Figure 3(c) shows the results of my experiment with DELPSI and DELEPS. Rather than solving for the rates of change of the baseline components, I solve for DELPSI and DELEPS for each baseline. That is, I solve for the orientation of the axis about which each baseline appears to be rotating (the "offset-axis" model). I also solve for the rate of change of baseline length for the two baselines, so that the number of parameters in the solution is the same as for the run which produced Figure 3(b). The RMS of the residuals for this run is 3.98 microns for the $\mathrm{SN}$ data shown (4.18 for the SE data). Thus, the goodness of fit is about the same as for the conventional run. If I omit the change-in-length parameter the fit degenerates slightly, to an RMS of 4.30 microns. The values of DELPSI and DELEPS indicate axes a few tenths of an arcsecond offset from nominal but are not the same for the two baselines. The values of DELPSI and DELEPS are quite well determined - the worst case differs from zero by 2.6 times its mean error - and remain the same if the change-inlength parameter is omitted. The value of the change-in-length parameter corresponds to that which can be computed from the conventional linear-drift run. In short, the scheme works.

I have run a similar experiment with data taken on 8 October 1987. For this data set, the offsetaxis model actually was slightly better for both baselines than the conventional linear-drift model. I should say that these data sets have not been pre- or post-selected in any way; they were chosen simply on the basis of the overall quality of the data and the strong signature of the baseline changes over the course of the night. I was actually quite surprised to see that, in practice, the two models were essentially equivalent, that is, for these data at least, modelling the baseline changes as a rotation about an offset axis is pretty much equivalent to modelling the changes as linear drifts with time. This is what we would expect, of course, from the geometry I have described in section 4, but I thought it important to verify the effect with real data. I do not claim that all optical interferometer data fit into this mold and I expect that neither model is entirely adequate.

What the offset-axis model provides is the baseline's axis of rotation within the reference frame of the star coordinates. If our baselines were tied to the Earth this would be quite valuable data, containing information both on large-scale rotations or distortions in the input star catalog and (accumulated over many years) on the validity of our models for Earth orientation and rotation. But without the assurance that the baseline's axis of rotation is that of the Earth, DELPSI and DELEPS do not provide us with any information that we can practically use. We have lost any well-defined 


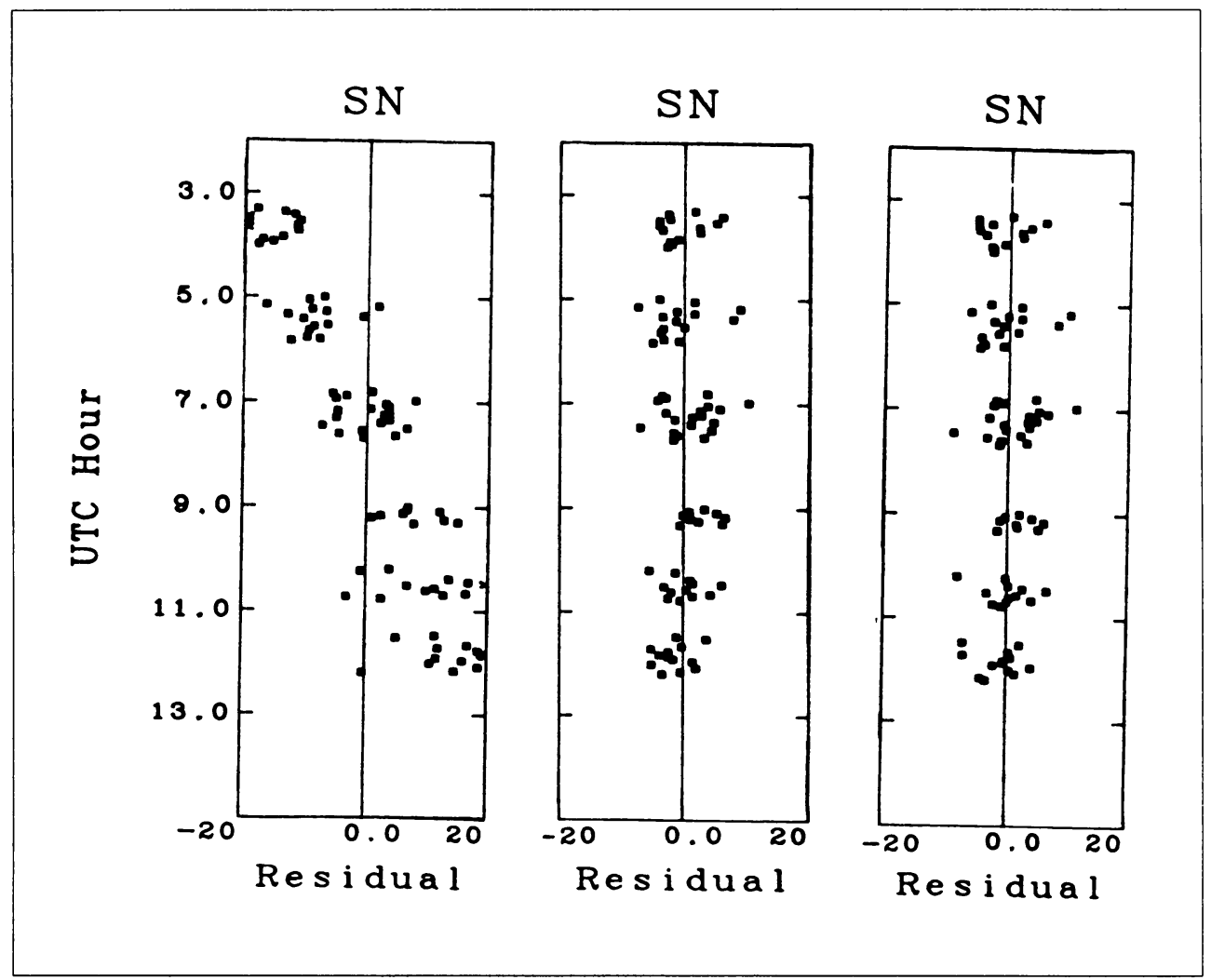

Figure 3. Interferometer path-length residuals, in microns, with various baseline motion models: (a) (left) no motion; (b) (center) linear drift of rectangular components; (c) (right) rotation about slightly offset axis

physical anchor for our coordinate system; we are reduced to trusting the input star catalog (the FK5) because our axis of rotation has no external significance.

We can always solve for star position corrections within the reference frame of the input star catalog. I put artificial errors in the catalog positions of three FK5 stars (numbers 52, 862, and 1619) by adding 0.20 arcsec to both coordinates of each. I can recover these errors from the 21 August data either from a solution based on the linear-drift model of baseline motion or a solution based on the offset-axis model. I then rotated the entire FK5 input catalog system (including the three star positions with the artificial errors) by 0.13 arcsecond and re-reduced the data. In the offset-axis model solution the DELPSI and DELEPS values adjusted themselves to reflect the additional rotation of the system. But the star positions corrections were unchanged, that is, they still indicated their original 0.20 arcsecond errors. Those are their errors within the rotated FK5 system, not their total errors. When I applied the linear-drift model solution, I obtained corresponding results - the rates of change of the baseline components adjusted themselves but the star position corrections were essentially unchanged. This is the point of this paper: as long as we have to solve for baseline motion 
from the observations themselves, we can recover star position corrections only within the system of the input catalog. Our observations are not fundamental.

\section{Conclusion}

In order to do fundamental astrometry, we must effectively "tie down" our baselines to the Earth in some well-defined way. Unfortunately, the siderostats and the piers they sit on must be exposed to many types of environmental influences. It is not realistic to expect baselines which are tens of meters long, exposed to the elements, to be stable to a few parts in $10^{8}$ in three dimensions over many hours. Accepting the fact that there will be significant changes in the baseline components during our observations, we need to measure these changes with respect to either (1) the Earth itself or (2) an astronomical reference system known a priori to be fundamental to a high degree of precision.

The new Naval Observatory astrometric interferometer now being designed will incorporate a complex laser metrology system to continuously measure the siderostat positions with respect to Earth-fixed benchmarks. Guaranteeing that these benchmarks, called optical anchors, are stable at the micron level is a challenge, but one with which the geodetic community has had experience. Jim Hughes and Don Hutter are engaged in the design work for the metrology system, which will rest on thermally isolated invar tripods driven into bedrock.

The other approach is to use a small number of stars whose positions and proper motions are very precisely known within an established fundamental system, and measure the baseline motion from their observations alone. Quasars with VLBI positions would be the best candidates, but the interferometer would have to operate efficiently beyond 15 th magnitude. We expect that the new Naval Observatory interferometer will be able to reach the brightest quasars, so we anticipate that this strategy will also be available to us.

We are entering an era when the most precise wide-angle astrometry - that performed from space platforms - will not be fundamental. It may be the case that the traditional Earth-based astronomical coordinates of right ascension and declination are actually not optimum for the very high precision astrometry of the future. There has already been much discussion in favor of abandoning the equinox as the origin of right ascension; perhaps this is only the beginning of the disassembly of the traditional fundamental astronomical reference system. Nevertheless, we should recognize that the need to relate terrestrial systems to astronomical systems will remain. The issues may be cast in a different terminology, but the basic scientific challenges remain. A small class of astronomical instruments -those which are capable of establishing what we now call fundamental systems - will remain the essential link between the sky and the Earth. With careful design, optical interferometers will soon be important members of this class.

\section{References}

Colavita, M.M., Shao, M., Staelin, D.H.: 1987, Appl. Opt. 26, 4113.

Kaplan, G.H., Hughes, J.A., Seidelmann, P.K., Smith, C.A., Yallop, B.D.: 1989, Astron. J. 97, 1197. Kaplan, G.H., Hershey, J.L., Hughes, J.A., Hutter, D.J., Johnston, K.J., Mozurkewich, D., Simon, R.S., Colavita, M.M., Shao, M., Hines, B.E., Staelin, D.H.: 1988, in Proceedings of the 
NOAO-ESO Conference on High Resolution Imaging by Interferometry, ed. F. Merkle (European Southern Observatory, Garching), p. 841.

Mozurkewich, D., Hutter, D.J., Johnston, K. ., Simon, R.S., Shao, M., Colavita, M.M., Staelin, D.H., Hines, B., Hershey, J.L., Hughes, J.A., Kaplan, G.H.: 1988, Astron. J. 95, 1269.

Shao, M.: 1988, in Proceedings of the NOAO-ESO Conference on High Resolution Imaging by Interferometry, ed. F. Merkle (European Southern Observatory, Garching), p. 823.

Shao, M., Colavita, M.M., Hines, B.E., Staelin, D.H., Hutter, D. ., Johnston, K.J., Mozurkewich, D., Simon, R.S., Hershey, J.L., Hughes, J.A., Kaplan, G.H.: 1988, Astron. Astrophys. 193, 357.

\section{Discussion}

KHARIN: (1) What is the diameter of the mirror at the siderostat and (2) what detectors were used?

KAPLAN: (1) The primary siderostat mirrors on Mt Wilson are $25 \mathrm{~cm}$ in diameter. However, the effective aperture of the instrument is only $8 \mathrm{~cm}$. (2) We detect interference by modulating the instrumental optical path length by about one wavelength at a frequency of $500 \mathrm{~Hz}$. We then slowly slew the delay line. When interference is achieved, it occurs only over a range in delay of about one wavelength because of the wide bandwidth. Therefore, we search for a $500 \mathrm{~Hz}$ modulation of the light in the combined beams, which indicates that the beams are interfering. We use standard cooled photomultiplier tubes for detection.

TREUHAFT: Is it practical to monitor the baseline motion as a function of temperature or by direct metrology?

KAPLAN: The baseline motion over the course of a night seems not to be generally repeatable from night to night, even though the Mt Wilson temperature function is. We have experimented with pier-to-pier metrology systems, but these monitor only distance (length), whereas our baseline motion is mostly in the form of a rotation.

MORRISON: (1) Is it your intention to publish a catalogue with the current Mt Wilson interferometer? (2) To what extent are the astrophysical and astrometric programmes complementary in the use of observation time? (3) How long will the current astrometric programme continue?

KAPLAN: (1) We are a little reluctant to publish a "catalog," as such, since (a) we can observe a relatively small number of stars; (b) we have only single-epoch positions; and (c) we do not yet fully understand our systematic errors. We have published (Mozurkewich et al. 1988) our observed offsets from FK5 positions based on one-color observations. Our two-color observations are much more precise, and we do plan to publish these results also.

(2) There is very little overlap — they are essentially independent observing programs.

(3) Undoubtedly until the new interferometer is built, in 1993.

BASTIAN: If among the stars reachable by your instrument there would be three radio objects, then you could check your results against independent measurements. Because there is nothing except radio data rivaling your accuracy, are there any such radio stars?

KAPLAN: I agree we are in the situation of having no good checks on our accuracy. The situation is somewhat like the early days of VLBI. HIPPARCOS observations would be very helpful. Our sky coverage and magnitude range is quite restricted with the current Mt Wilson interferometer, and there are not enough radio stars in our observing range to make a meaningful check. 